\title{
Twos in human visual perception
}

\author{
Liuba Papeo \\ Institut des Sciences Cognitives-Marc Jeannerod, UMR5229, Centre National de la \\ Recherche Scientifique (CNRS) and Université Claude Bernard Lyon 1, 67 Bd. Pinel \\ - 69675 Bron (France) \\ *Correspondence: Liuba.papeo@isc.cnrs.fr
}

Keywords: face perception; body perception; perceptual grouping; spatial cognition; social cognition; social interaction. 


\begin{abstract}
Human vision serves the social function of detecting and discriminating with high efficiency conspecifics and other animals. The social world is made of social entities as much as the relations between those entities. Recent work demonstrates that vision encodes visuo-spatial relations between bodies with the same efficiency and high specialization of face/body perception. Specifically, perception of face-to-face (vs. non-facing) bodies evokes effects compatible with the most robust markers of face-specificity such as the behavioral inversion effect and increased activity in selective visual areas. Another set of results suggests that face-to-face bodies are processed as a grouped unit, analogously to facial features in a face. The facing dyad in the visual cortex may be the earliest rudimentary representation of social interaction.
\end{abstract}




\section{Sociality shapes perception}

A decade ago, vision scientist, Ken Nakayama, announced that vision science was going social (Nakayama, 2011). The social function of vision appears clear from the highly specialized mechanisms for face and body perception. Embracing the idea that vision has been massively shaped by the requirements of social life, Nakayama anticipated the discovery of perceptual adaptations for other aspects of the social world.

Faces and bodies are the physical actors of social life; but social life is only realized through the relations between those entities. In sum, faces and bodies are socially relevant in that they carry the potential to realize social relations. Novel research is asking whether the visual system is sensitive to socially relevant spatial relations as much as to social entities. A challenge for this field is to single out visual perception of (relations between) multiple bodies from more abstract, conceptual or affective representations of social scenarios. To this end, the current focus is on studies that have targeted physiological measures of activity in visual cortex, or performance-based measures of perception, in which performance reflects how the subjects perceive the stimuli (e.g., visual search or near-threshold stimulus detection and recognition; see Scholl \& Gao, 2013). This focus leaves aside the rich corpus of studies that have addressed the processing social (multiple-body) scenarios in the social brain and other networks, outside the visual cortex (for reviews see Hari \& Kujala, 2009; Quadflieg \& Koldewyn, 2017), or through the subjects' perceptual judgments, which capture inferential processes beyond perception (for a discussion of the distinction between perception and judgment, see Firestone \& Scholl, 2016). 
In many instances of physical or communicative exchanges, people are close and face-to-face, a positioning that favors fundamental social processes such as gaze following and shared attention (Baron-Cohen, 1997; Birmingham \& Kingstone, 2009). Recent findings show that face-to-face body dyads are processed as single attentional/perceptual units, yielding processing signatures that echo robust markers of the highly specialized face perception process. This research is revealing uncharted functions of vision and the building blocks of social cognition.

\section{Visual tuning for facing dyads}

A face is attended to, detected and discriminated in terms of identity much better than an inverted face (e.g., Bruyer, 2011; Jiang, Costello, \& He, 2007; Purcell \& Stewart, 1988; Rezlescu, Susilo, Wilmer, \& Caramazza, 2017). The cost of inversion, or face inversion effect (FIE), is higher for faces than for other familiar objects. The FIE denotes neural tuning to the face configuration, which makes perception particularly efficient, though particularly susceptible to spatial perturbation. Efficiency would result from a perceptual mechanism, so-called configural, that readily represents the face as a whole and relies on spatial relations between parts for recognition and discrimination across exemplars (Carey, 1992; Collishaw \& Hole, 2000; Maurer, Le Grand, \& Mondloch, 2002; Rossion \& Gauthier, 2002). The inversion effect has been generalized to single bodies that, like faces, are familiar, multi-part objects with dedicated brain areas (Reed, Stone, Bozova, \& Tanaka, 2003; Reed, Stone, Grubb, \& McGoldrick, 2006; Stein, Sterzer, \& Peelen, 2012). 
Recent work shows an inversion effect for dyads of bodies facing each other. With stimuli presented around perceptual threshold and masked in a visual-categorization task (i.e., deciding whether a stimulus featured bodies or other objects), inversion disrupts detection of facing dyads more than of nonfacing dyads (Figure 1) (Papeo, Stein, \& Soto-Faraco, 2017). The two-body inversion effect (2BIE), the larger cost of inversion for facing vs. non-facing dyads, appears the result of the mere relative positioning, defined by the body, even without information about head/gaze direction (e.g., in bodies with blurred head-area; Papeo \& Abassi, 2019), or by the head alone (Strachan, Sebanz, \& Knoblich, 2019), irrespective of the semantic content or coherence of the implied interaction (Papeo et al., 2017). The 2BIE does not generalize to human-object or object-object pairs (Papeo \& Abassi, 2019). Extending the knowledge gained on face/body perception, the 2BIE suggests visual tuning to a unitary configuration of two bodies facing each other as if interacting.

\section{Attentional advantage of facing dyads}

The interpretation of the inversion effect as an index of visual tuning to face and body configurations meshes well with the ability of those stimuli to recruit attention more strongly than other objects, as documented with, among others, visual search and change detection tasks (Cohen, Alvarez, Nakayama, \& Konkle, 2017; Downing, Bray, Rogers, \& Childs, 2004; Hershler \& Hochstein, 2005; New, Cosmides, \& Tooby, 2007). Recently, an attentional advantage has been reported for facing body dyads, over non-facing, but otherwise identical, dyads. 
In a visual search task, in which participants search for a target among distractors, participants saw arrays of dyads where target and distractors were defined by the spatial relation between two bodies: the target was either a facing dyad surrounded by non-facing dyads (distractors), or a non-facing dyad (two bodies facing away from each other) surrounded by facing dyads (distractors). With fast presentation of the arrays, participants were more likely to detect a facing dyad among non-facing dyads, than a non-facing dyad among facing dyads. The search asymmetry reflects how well the targets could recruit attention: facing dyads did so more strongly than non-facing dyads (Papeo, Goupil, \& SotoFaraco, 2019; see also Vestner, Tipper, Hartley, Over, \& Rueschemeyer, 2019; Vestner, Gray, \& Cook, 2020).

Humans can efficiently search scenes by spatial location, visual features, or whole objects. We now know that human attention also benefits from fast appraisal of spatial relations between individuals in crowds, and uses that information to give priority to face-to-face bodies.

\section{Neural tuning and representational sharpening}

Compared with their scrambled version or other familiar objects, faces and bodies increase activity in specific areas of the visual cortex. A recent functional magnetic resonance imaging study shows that face- and body-selective cortices also respond to facing bodies more strongly than to identical but not facing bodies (Abassi \& Papeo, 2020; Figure 1). The same brain areas do not distinguish between interacting and noninteracting people based on contextual cues (e.g., accessories or clothing) or action knowledge (Quadflieg, Gentile, \& Rossion, 
2015), which suggests encoding of basic visuo-spatial cues of interaction (i.e., orientation of head/body, and possibly others such as limb position and body lean; see Hafri, Papafragou, \& Trueswell, 2013; Hafri, Trueswell, \& Strickland, 2018; Zhou, Han, Liang, Hu, \& Kuai, 2019), as opposed to more abstract representation of interaction. The recruitment of visual areas, whose functioning has been linked to the special way in which faces and bodies are processed, strengthens the analogy between face/body and facing-dyad perception suggested by the above behavioral effects.

The study also shows that seeing bodies in facing ( $v s$. non-facing) dyads enhances, or sharpens, the representation of bodies in visual cortex, as indexed by reliably distinct neural patterns across different body postures. Representational sharpening, previously reported for non-body objects seen in their typical visual context (e.g., a car on the road; Brandman \& Peelen, 2017), is taken to reveal internal expectations about the visual environment (Heilbron, Richter, Ekman, Hagoort, \& de Lange, 2020). Accordingly, a facing dyad would match an internal representation, which reflects how the visual system expects two nearby bodies to be. 

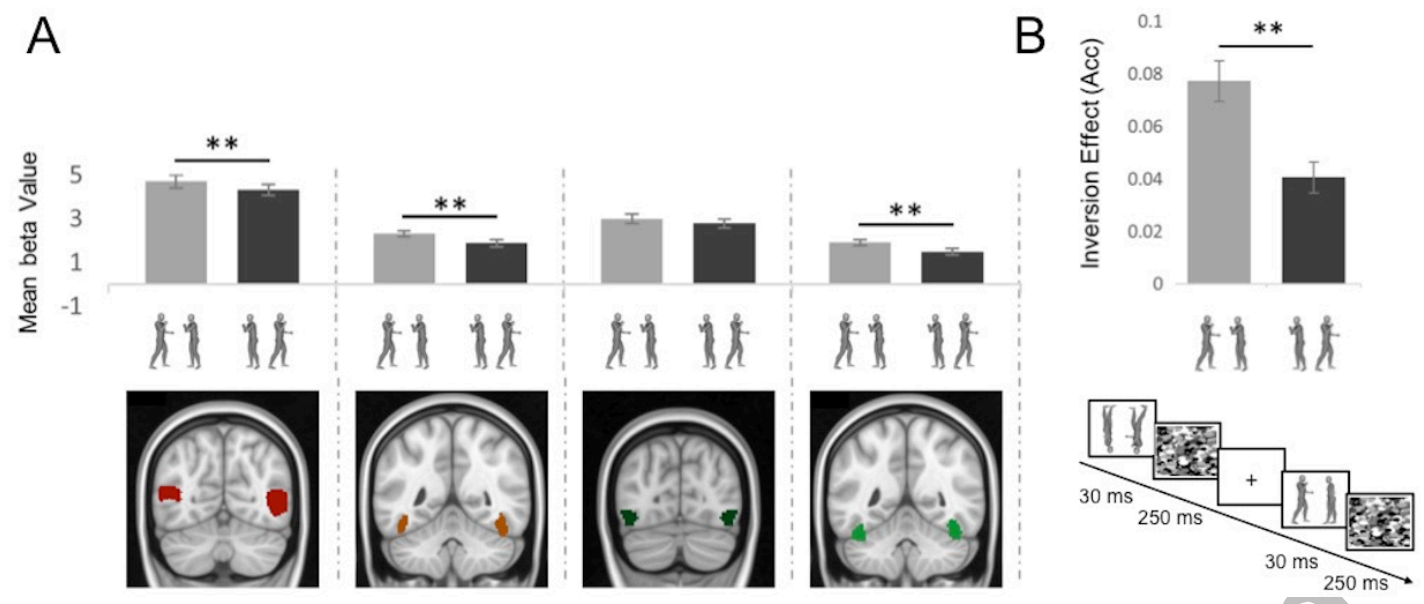

Figure 1. Markers of perceptual specialization for facing body dyads. (A) Increased fMRI response (mean beta value) for facing and non-facing body dyads in face- and body-selective visual brain areas (extrastriate body cortex in red, fusiform body area in orange, occipital face area in green; fusiform face area in light green). (B) The behavioral inversion effect (difference in the proportion of correct responses to upright minus inverted stimuli) for facing and non-facing body dyads, measured during a visual categorization task (i.e. reporting whether each image featured bodies or chairs) with short presentation and backward masking of the stimuli. ${ }^{* *}$ denote statistically significant differences between two conditions. Data reproduced from Abassi \& Papeo (2010).

\section{Grouping of bodies: Effects of access versus encoding}

Faster/better stimulus detection and recognition of stimuli encompassing multiple parts (or objects) has often been linked to perceptual grouping, that is, the representation of parts as a structure unit, or a group. Perceptual grouping is invoked across different levels of vision and types of stimuli: It applies to dots and lines in gestalt perception, as well as to facial features in a face, and parts in a body -although the relationship between gestalt-like grouping and configural processing, in the context of face/body perception, remains unclear (Pomerantz \& Portillo, 2011; Rezlescu, Susilo, Wilmer, \& Caramazza, 2017). Is grouping a candidate mechanism for the efficient processing of facing dyads? 
A signature of grouping for faces is delayed access to internal facial features, called object-inferiority effect (Suzuki \& Cavanagh, 1995): thus, searching for a face is more efficient than searching for a component within it, such as the mouth. Thus, while grouping yields efficient processing of the grouped configuration, it has a cost in terms of access to the component parts. Echoing the object-inferiority effect, it has been shown that, in visual search through a crowd, it takes longer to detect a target-body when in a facing dyad than when in a non-facing dyad.

On the other hand, effects of neural sharpening (Abassi \& Papeo, 2020) and recognition performance (Vestner et al., 2019) show enhanced representation of single bodies in facing ( $v s$. non-facing) dyads, just like of facial features (e.g., nose) in faces vs. scrambled faces, sometimes called objectsuperiority effect (Homa, Haver \& Schwartz, 1976). Despite the apparent contradiction, the object-inferiority and object-superiority effects capture different processing stages (Figure 2). The former, found in visual search, concerns access, and shows that the whole (a dyad) is accessed earlier than its component parts (single bodies). The latter informs on the strength of representation. In sum, analogously to facial features, single bodies in facing dyads are accessed after the whole to which they belong, but are represented better than the same bodies in non-facing dyads. 
faces
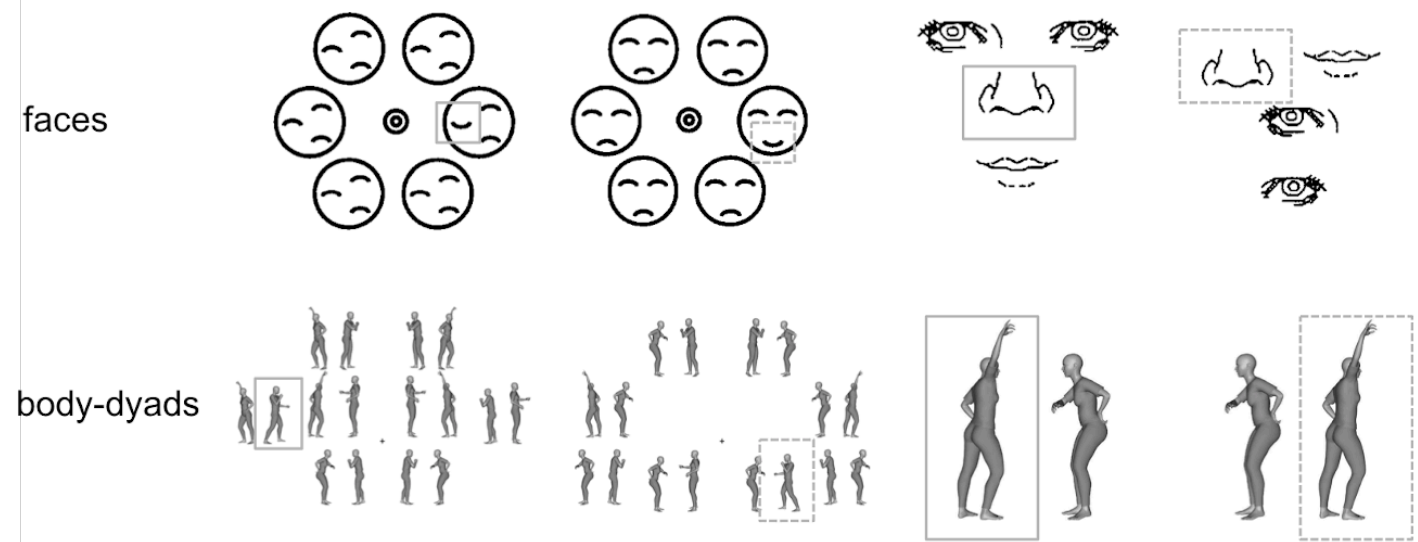

Figure 2. Effects of access vs. encoding of faces and facing dyads. Objectinferiority effects in visual search: the only upward arc is detected faster when in a meaningless configuration than when in a face configuration (figure reproduced from Suzuki \& Cavanagh, 1995); with fast stimulus presentation, a target body (e.g., the only "punching" body) is more likely to be detected when in a non-facing dyad than when in a facing dyad (Papeo et al., 2019). Objectsuperiority effects in individuation tasks: a nose is individuated better, among other noses, when first seen in a face than in a scrambled face; a single body is individuated better when first seen in a facing dyad than in a non-facing dyad (Vestner et al., 2019). Lines around figure elements denote targets (full and dashed lines for targets associated with better and lower performance, respectively).

\section{Multiple-body versus multiple-object perception}

Markers of face specificity, such as attentional advantage, inversion effect, and increased activity in visual areas specifically linked to configural processing, suggest analogous visual specificity for facing dyads. But, are multiple bodies (or dyads, at least) special relative to other multiple-object sets?

Object pairs in regular, meaningful configurations (e.g., a lamp above a table) are recognized particularly efficiently, suggesting that they too might be processed as a grouped unit (Kaiser, Quek, Cichy, \& Peelen, 2019). However, no other effect, common to faces, bodies and facing dyads, has been reported for 
non-body object pairs (Table 1), suggesting different underlying mechanisms for object pairs and body dyads. In effect, regular-object pairs recruit brain areas functionally and anatomically dissociated from the visual areas involved in facing-dyad perception. Effects of objects presented in regular configurations or in functional (i.e., convenient to use) configurations (e.g., a pen on top of a notebook) have been reported in areas such as the parahippocampal place area (Kaiser, Stein, \& Peelen, 2014) and the object-general lateral occipital cortex (Baeck, Wagemans, \& de Beeck, 2013; Roberts \& Humphreys, 2010), respectively.

Moreover, alleged grouping of multiple objects captures the effect of statistical regularities (i.e., typical absolute or relative object locations) and semantic relations in visual perception. Neither factor promises to offer an account of facing-dyad perception (Abassi \& Papeo, 2010; Papeo et al., 2017; Quadflieg et al., 2015). The working hypothesis for facing dyads is that they are face-like special, perhaps because of their biological relevance, and despite the facts that they might not be more recurrent than other multiple-body configurations in the visual world, and do not need to represent semantically coherent relations to be processed efficiently in visual cortex. 
Table 1. Effects in visual perception of faces, body dyads and regular-object pairs (reported $=\mathrm{R}$; not reported $=\mathrm{nR}$ ).

\begin{tabular}{|c|c|c|c|c|}
\hline & \multicolumn{2}{|c|}{$\begin{array}{l}\text { Face } v s \text {. scrambled } \\
\text { face or other objects }\end{array}$} & \multicolumn{2}{|c|}{$\begin{array}{cc}\begin{array}{c}\text { Facing } v s . \text { non-facing } \\
\text { dyads }\end{array} & \text { Regular vs. irregular } \\
\text { object pairs }\end{array}$} \\
\hline & $\begin{array}{l}\text { 10 } \\
2 b \\
\approx\end{array}$ & $\begin{array}{c}d y \approx \\
0 \\
0\end{array}$ & 28 & th \\
\hline $\begin{array}{l}\text { Stronger recruitment of } \\
\text { attention } R\end{array}$ & $\mathrm{I}$ & $\mathrm{R}$ & $\mathrm{R}$ & $\mathrm{nR}$ \\
\hline Increased Inversion Effect & $\mathrm{r}$ & $\mathrm{R}$ & $\mathrm{R}$ & 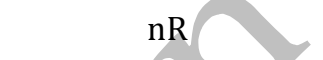 \\
\hline $\begin{array}{l}\text { Recruitment of areas for } \\
\text { configural processing }\end{array}$ & $\mathrm{I}$ & $\mathrm{R}$ & $\mathrm{R}$ & \\
\hline $\begin{array}{l}\text { Efficient recognition } \\
\text { (faster/more accurate) }\end{array}$ & I & $\mathrm{R}$ & $\mathrm{R}$ & $\mathrm{R}$ \\
\hline Object-inferiority effect & $\mathrm{r}$ & $\mathrm{R}$ & $\mathrm{R}$ & $\mathrm{nR}$ \\
\hline Object-superiority effect & $\mathrm{I}$ & $\mathrm{R}$ & & $\mathrm{nR}$ \\
\hline
\end{tabular}

\section{Conclusions}

Research on multiple-body perception suggests that spatial relations among objects matter in visual perception, so much that a body is represented in profoundly different ways depending on whether or not it faces toward another, and is reciprocated. If it does, a new attentional/perceptual unit is formed, larger and more complex than a single object.

This research defines a new class of stimuli that, I propose, can be better described and understood by analogy with faces. Like faces, facing dyads form a homogeneous class of multiple-part objects, where members share the general arrangement of parts (two bodies face-to-face), and are discriminated based on more subtle variations in shape and spatial relations between parts. Although the relationship between the face/body perception and multiple-body (dyad) 
perception remains unclear, the analogy between the two cases proves productive, as the extensive research on face perception guides and inspires the younger field of multiple-body perception. Moreover, the analogy is not merely conceptual: perception of facing dyads suggests grouping of multiple bodies into a whole, whose processing yields effects compatible with the most robust markers of face-specificity.

Thus, facing dyads would be as special to human vision and faces. Why so? As important as detecting and discriminating animate entities, is detecting and discriminating social interactions, in order to extract information about social agents, their relationships and social conducts. Being common to many social events, facingness -the mutual perceptual accessibility of agents- is a redundant feature of the social world, which might call for efficient coding in dedicated brain structures.

Previous seminal work has described perceptual adaptations for identifying, with high efficiency, biologically relevant aspects of the world that serve as input for domain-specific cognitive operations (Carey, 2011). In this perspective, the visual representation of facing dyads could act as a building block for inference on social interaction. Growing evidence suggests the existence of a domain-specific brain network for understanding dyadic social interactions (Isik, Koldewyn, Beeler, \& Kanwisher, 2017; Walbrin, Downing, \& Koldewyn, 2018; see also Wurm \& Caramazza, 2019; Wurm, Caramazza, \& Lingnau, 2017), possibly with a homologous in the non-human primate brain (Sliwa \& Freiwald, 2017). Across the available studies, facingness appears as a key, reliable signal to recruit that network. The facing dyad in the visual cortex may be the earliest rudimentary representation of a social interaction. 


\section{Acknowledgments}

The author is grateful to Jean-Rémy Hochmann and Alfonso Caramazza for valuable discussion on an early version of this article. This work was supported by the European Research Council (Project THEMPO, Starting Grant 758473). 


\section{References}

Abassi E, Papeo L: The representation of two-body shapes in the human visual cortex. J Neurosci 2019, 40:852-863.

Baeck A, Wagemans J, de Beeck HPO: The distributed representation of random and meaningful object pairs in human occipitotemporal cortex: the weighted average as a general rule. Neuroimage 2013, 70:37-47.

Baron-Cohen S: How to build a baby that can read minds: Cognitive mechanisms in mindreading. The maladapted mind: Classic readings in evolutionary psychopathology, 1997, 207-239.

Birmingham E, Kingstone A: Human social attention. Ann N Y Acad Sci 2009, 1156:118-140.

Brandman T, Peelen MV: Interaction between Scene and Object Processing Revealed by Human fMRI and MEG Decoding. J Neurosci 2017, 37:77007710.

Bruyer R. Configural face processing: A meta-analytic survey. Perception 2011, $40: 1478-1490$

Carey S: Becoming a face expert. Philos Trans R Soc Lond B Biol Sci 1992, $335: 95-103$

Carey S: Concept innateness, concept continuity, and bootstrapping. Behav Brain Sci 2011, 34:152-162.

Cohen MA, Alvarez GA, Nakayama K, Konkle T. Visual search for object categories is predicted by the representational architecture of high-level visual cortex. J Neurophysiol 2017, 117:388-402.

Collishaw SM, Hole GJ. Featural and configurational processes in the recognition of faces of different familiarity. Perception 2000, 29:893-909. 
Downing PE, Bray D, Rogers J, Childs C: Bodies capture attention when nothing is expected. Cognition 2004, 93:B27-B38.

Firestone C, Scholl BJ. Cognition does not affect perception: Evaluating the evidence for "top-down" effects. Behav Brain Sci 2016, 39:e229.

Hafri A, Papafragou A, Trueswell JC: Getting the gist of events: Recognition of two-participant actions from brief displays. J Exp Psychol Gen 2013, 142:880.

Hafri A, Trueswell JC, Strickland B: Encoding of event roles from visual scenes is rapid, spontaneous, and interacts with higher-level visual processing. Cognition 2018, 175:36-52.

Hari R, Kujala MV (2009). Brain basis of human social interaction: from concepts to brain imaging. Physiol Rev 89:453-479.

Heilbron M, Richter D, Ekman M, Hagoort P, de Lange FP. Word contexts enhance the neural representation of individual letters in early visual cortex. Nat Commun 2020, 11:1-1.

Hershler 0, Hochstein S. At first sight: A high-level pop out effect for faces. Vision Res 2005, 45:1707-1724.

Homa D, Haver B, Schwartz T: Perceptibility of schematic face stimuli. Evidence for a perceptual Gestalt. Mem Cognit 1976, 4:176-185.

Isik, L., Koldewyn, K., Beeler, D., and Kanwisher, N. (2017) Perceiving social interactions in the posterior superior temporal sulcus. Proc. Natl. Acad. Sci. 114, 9145-9152.

Jiang Y, Costello P, He S. Processing of invisible stimuli: Advantage of upright faces and recognizable words in overcoming interocular suppression. Psychol Sci 2007, 18:349-355. 
Kaiser D, Quek GL, Cichy RM, Peelen MV. Object vision in a structured world. Trends Cogn Sci 2019.

Kaiser D, Stein T, Peelen MV: Object grouping based on real-world regularities facilitates perception by reducing competitive interactions in visual cortex. Proc Natl Acad Sci 2014, 111:11217-22.

Kuhlmeier, pp. 197-230. MIT Press.

Maurer D, Le Grand R, Mondloch CJ. The many faces of configural processing. Trends Cogn Sci 2002, 6:255-260.

Nakayama, K. (2011). Introduction: Vision going social. In R. B. Adams, N.

Ambady, K. Nakayama \& S. Shimojo (Eds.), The Science of Social Vision, Oxford

New J, Cosmides L, Tooby J: Category-specific attention to animals reflects ancestral priorities, not expertise. Proc Natl Acad Sci 2007, 104:1659816603.

Papeo L, Abassi E: Seeing social events: the visual specialization for dyadic human-human interactions. J Exp Psychol Hum Percept Perform 2019, $45: 877-888$.

Papeo L, Goupil N, Soto-Faraco S: Visual search for people among people. Psychol Sci 2019, 30:1483-1496.

Papeo L, Stein T, Soto-Faraco S: The two-body inversion effect. Psychol Sci 2017, 28:369-379.

Pomerantz JR, Portillo MC: Grouping and emergent features in vision: Toward a theory of basic Gestalts. J Exp Psychol Hum Percept Perform, 37:13311349. 
Purcell, D. G., and Stewart, A. L. (1988). The face-detection effect: Configuration enhances detection. Perception \& Psychophysics, 43, 355-366.

Quadflieg S, Gentile F, Rossion B: The neural basis of perceiving person interactions. Cortex 2015, 70:5-20.

Quadflieg S, Koldewyn K: The neuroscience of people watching: how the human brain makes sense of other people's encounters. Ann N Y Acad Sci 2017, 1396:166-182.

Reed CL, Stone V, Bozova S, Tanaka J. The body inversion effect. Psychol Sci 2003, 302-308.16.

Reed CL, Stone V, Grubb JD, McGoldrick JE: Turning configural processing upside down: part and whole body postures. J Exp Psychol Hum Percept Perform 2006, 32:73-87.

Rezlescu C, Susilo T, Wilmer JB, Caramazza A: The inversion, part-whole, and composite effects reflect distinct perceptual mechanisms with varied relationships to face recognition. J Exp Psychol Hum Percept Perform $2017,43: 1961$.

Roberts KL, Humphreys GW: Action relationships concatenate representations of separate objects in the ventral visual system. NeuroImage 2010, 52:15411548

Rossion, B., \& Gauthier, I. (2002). How does the brain process upright and inverted faces?. Behavioral and cognitive neuroscience reviews, 1(1), 6375.

Scholl, B. J. \& Gao, T. (2013) Perceiving animacy and intentionality: Visual processing or higher-level judgment? In: Social perception: Detection and 
interpretation of animacy, agency, and intention, ed. M. D. Rutherford \& V. A.

Sliwa J, Freiwald WA: A dedicated network for social interaction processing in the primate brain. Science 2017, 356:745-749.

Stein T, Sterzer P, Peelen MV: Privileged detection of conspecifics: Evidence from inversion effects during continuous flash suppression. Cognition 2012, 125:64-79.

Strachan JW, Sebanz N, Knoblich G: The role of emotion in the dyad inversion effect. PloS One 2019,14:7.

Suzuki S, Cavanagh P: Facial organization blocks access to low-level features: An object inferiority effect. J Exp Psychol Hum Percept Perform 1995, 21:901-913.

University Press.

Vestner T, Gray KL, Cook R: Why are social interactions found quickly in visual search tasks?. Cognition 2020, 200:104270.

Vestner T, Tipper SP, Hartley T, Over H, Rueschemeyer SA: Bound together: Social binding leads to faster processing, spatial distortion, and enhanced memory of interacting partners. J Exp Psychol Gen 2019, 148:1251-1268. Walbrin J, Downing P, Koldewyn K: Neural responses to visually observed social interactions. Neuropsychologia 2018, 112:31-39.

Wurm MF, Caramazza A, Lingnau A. Action categories in lateral occipitotemporal cortex are organized along sociality and transitivity. J Neurosci 2017, $37: 562-575$ 
Wurm MF, Caramazza A: Lateral occipitotemporal cortex encodes perceptual components of social actions rather than abstract representations of sociality. NeuroImage 2019, 202:116153.

Zhou C, Han M, Liang Q, Hu YF, Kuai SG. A social interaction field model accurately identifies static and dynamic social groupings. Nature Hum Behav 2019, 3:847-55. 